\title{
Subdiagnóstico de la lesión renal aguda en pacientes obstétricas complicadas en la Unidad de Cuidados Intensivos
}

Underdiagnosis of acute kidney injury in complicated obstetric patients in the Intensive Care Unit

Subdiagnóstico da lesão renal aguda em pacientes obstétricas complicadas na Unidade de Terapia Intensiva

\author{
Alfonso Estrada-Gutiérrez, ${ }^{*, \neq}$ César Maya-Contreras, ${ }^{*}$ Themis Gwendolyne Aguilar-Arciga, ${ }^{*}$ \\ Angélica García-Gómez,§ Pedro Barriga-Ferreyra*,‡
}

\begin{abstract}
RESUMEN
Objetivo: Definir la prevalencia y factores asociados de lesión renal aguda en el embarazo en la Unidad de Cuidados Intensivos del Hospital de la Mujer, Morelia, Michoacán, México.

Material y métodos: Estudio retrospectivo, transversal y descriptivo de enero de 2013 a agosto de 2018. Pacientes: 213 expedientes de pacientes obstétricas complicadas. Criterios de inclusión: pacientes obstétricas complicadas que ingresaron a la $\mathrm{UCl}$, pacientes que cumplieron criterios para lesión renal aguda. Criterios de exclusión: pacientes con lesión renal crónica, expediente clínico no disponible. Sólo 154 cumplieron con los criterios de selección.

Resultados: Se incluyeron 154 pacientes obstétricas complicadas; un promedio de $25.6 \mathrm{p} \pm 1.6$ por año. Treinta y seis por ciento tuvo diagnóstico de eclampsia; $35.3 \%$ preeclampsia; $29.3 \%$ síndrome de HELLP; $19.3 \%$ hemorragia obstétrica, $10 \%$ sepsis. Se demostró asociación de PR-AKI con síndrome de $\operatorname{HELLP}(p=0.0003)$ y preeclampsia $(p=0.01)$.

Se encontró un subdiagnóstico de $36.7 \%$ al buscar PR-AKI utilizando los criterios RIFLE y AKI $(p=0.000007)$. De las pacientes con PR-AKI grado 3 , sólo $20 \%$ requirió terapia de reemplazo renal continua.

Conclusiones: La lesión renal asociada al embarazo complicado tiene una prevalencia de 6.7\%. Las complicaciones asociadas a PR-AKI son síndrome de HELLP y preeclampsia. La PR-AKI está subdiagnosticada hasta en $36.7 \%$.

Palabras clave: Lesión renal aguda, terapia de reemplazo renal, unidad de cuidados intensivos, eclampsia, preeclampsia, síndrome de HELLP.
\end{abstract}

\section{ABSTRACT}

Objective: To define the prevalence and associated factors of acute renal injury in pregnancy (PR-AKI) in the Intensive Care Unit (ICU) of the Women's Hospital, Morelia, Michoacán, Mexico.

Material and methods: Retrospective, cross-sectional and descriptive study from January 2013-August 2018. Ambit: Intensive Care Unit of the Women's Hospital. Patients: We reviewed 213 files of complicated obstetric patients. Inclusion criteria: complicated obstetric patients that merit admission to the ICU, patients who met criteria for LRA. Exclusion criteria: patients with chronic.

Main variables of interest: Complicated obstetric patients (preeclampsia, eclampsia, HELLP syndrome, hemorrhagic shock and sepsis). It was investigated if they developed PR-AKI.

Results: 154 complicated obstetric patients were included; an average of $25.6 p$ \pm 1.6 per year. $36 \%$ had a diagnosis of eclampsia; $35.3 \%$ pre-eclampsia; $29.3 \%$ HELLP syndrome; $19.3 \%$ obstetric hemorrhage, $10 \%$ sepsis. Association of $P R$-AKI with HELLP syndrome $(p=0.0003)$ and pre-eclampsia $(p=0.01)$ was demonstrated.

A subdiagnosis of $36.7 \%$ was found when searching for PR-AKI using the RIFLE and AKI criteria $(p=0.000007)$. Of the patients with PR-AKI grade 3 , $20 \%$ required continuous renal replacement therapy.

\footnotetext{
* Hospital de la Mujer.

‡ Hospital Innova Médica.

$\S$ Hospital General «Dr. Miguel Silva».

Michoacán, México.
}

Recibido: 09/10/2020. Aceptado: 28/10/2020.

Citar como: Estrada-Gutiérrez A, Maya-Contreras C, Aguilar-Arciga TG, García-Gómez A, Barriga-Ferreyra P. Subdiagnóstico de la lesión renal aguda en pacientes obstétricas complicadas en la Unidad de Cuidados Intensivos. Med Crit. 2021;35(2): 79-83. https:// dx.doi.org/10.35366/99527

www.medigraphic.com/medicinacritica
Conclusions: Renal injury associated with complicated pregnancy has a prevalence of $6.7 \%$. The complications that most develop PR-AKI are HELLP syndrome and preeclampsia. PR-AKI is underdiagnosed.

Keywords: Acute kidney injury, renal replacement therapy, intensive care unit, eclampsia, preeclampsia, HELLP syndrome.

\section{RESUMO}

Objetivo: Definir a prevalência e os fatores associados à Lesão Renal Aguda na gravidez na Unidade de Terapia Intensiva do Hospital de la Mujer, Morelia, Michoacán, México.

Material e métodos: Estudo retrospectivo, transversal e descritivo de janeiro de 2013 a agosto de 2018. Pacientes: 213 prontuários obstétricos complicados. Critérios de inclusão: pacientes obstétricas complicadas admitidas na UTI, pacientes que preencheram os critérios para Lesão Renal Aguda. Critérios de exclusão: pacientes com Lesão Renal Crônica, ficha clínica não disponível. Apenas 154 atenderam aos critérios de seleção.

Resultados: 154 pacientes obstétricas complicadas foram incluídas; uma média de $25.6 p \pm 1.6$ por ano. $36 \%$ tiveram diagnóstico de eclâmpsia; $35.3 \%$ pré-eclâmpsia; $29.3 \%$ síndrome HELLP; $19.3 \%$ hemorragia obstétrica, $10 \%$ sepse. Foi demonstrada uma associação de PR-AKI com síndrome HELLP ( $p=$ $0.0003)$ e pré-eclâmpsia $(p=0.01)$.

Foi encontrado subdiagnóstico de $36.7 \%$ na busca de PR-AKI pelos critérios RIFLE e AKI $(p=0.000007)$. Dos pacientes com PR-AKI grau 3, $20 \%$ necessitaram de terapia de reposição renal contínua.

Conclusões: Lesões renais associadas à gravidez complicada apresentam prevalência de $6.7 \%$. As complicações associadas à PR-AKI são a síndrome HELLP e a pré-eclâmpsia. PR-AKl é subdiagnosticado em até $36.7 \%$.

Palavras-chave: Lesão renal aguda, terapia renal substitutiva, Unidade de Terapia Intensiva, eclâmpsia, pré-eclâmpsia, síndrome HELLP.

\section{INTRODUCCIÓN}

La lesión renal aguda en el embarazo (PR-AKI) es un síndrome de etiología multifactorial que se caracteriza por la disminución abrupta de la filtración glomerular: el riñón resulta incapaz de mantener la homeostasis de líquidos y electrolitos, reteniendo así productos nitrogenados (urea y creatinina). Se producen cambios hemodinámicos y vasculares en el embarazo normal, aumentando la tasa de filtrado glomerular (TFG) de 40-50\%. Dichos cambios inician durante el primer mes y persisten hasta el tercer trimestre. Por el contrario, la producción de creatinina se mantiene constante. ${ }^{1}$

La prevalencia e incidencia de PR-AKI varía de acuerdo al desarrollo socioeconómico de los países en rangos que abarcan de $4.2-15 \%$ y de $1 / 2,000-1 / 5,000$ embarazos respectivamente. ${ }^{2}$

Muchos factores contribuyen a estas variaciones: falta de criterios de definición uniformes, cambios fisiológicos en el embarazo que afectan la interpretación de 
las pruebas de laboratorio y diferencias regionales en los factores que contribuyen a la PR-AKI. Además, la lesión renal aguda asociada al embarazo a menudo no se reconoce hasta que es grave.

Respecto a su etiología, se conoce que en países desarrollados la principal causa de la PR-AKI es la eclampsia, mientras que en países en desarrollo es la sepsis. ${ }^{3}$ La PR-AKI constituye un reto diagnóstico y de tratamiento para los obstetras, nefrólogos e intensivistas.

Se han propuesto dos sistemas de clasificación para medir el daño renal: los criterios «AKIN» (clasificación para el daño renal agudo) y los criterios «RIFLE», que incluyen cinco categorías (riesgo, lesión, fracaso, pérdida y fase final). Ambas tienen una alta tasa de detección de LRA (38.5 y 28.5\%) respectivamente, que determina la severidad según los incrementos porcentuales de creatinina sérica y la disminución del gasto urinario. ${ }^{4}$

Los estudios obstétricos más recientes han comenzado a utilizar las escalas RIFLE y AKIN para determinar la lesión renal aguda en este grupo de pacientes. ${ }^{5,6}$ Sin embargo, los estudios de PR-AKI se realizan en otros países, por lo que la información sobre esta patología en México continúa siendo insuficiente. Se llevó a cabo este estudio con el fin de determinar la prevalencia de PR-AKI entre las mujeres atendidas en la Unidad de Cuidados Intensivos del Hospital de la Mujer de Morelia, Michoacán, México.

\section{MATERIAL Y MÉTODOS}

Diseño de la investigación: estudio cuantitativo, retrospectivo, transversal, observacional y descriptivo.

Protocolo: se recabaron las bases de datos del Hospital de la Mujer, Michoacán, México. Se revisaron en las bitácoras de la unidad de cuidados intensivos (UCI) los ingresos y egresos de pacientes con complicaciones obstétricas durante el periodo comprendido del 01 enero de 2013 al 01 de agosto de 2018 con diagnóstico de embarazo o puerperio complicado.

Se realizó un muestreo no probabilístico por conveniencia. Se incluyeron todas las pacientes obstétricas complicadas que ameritaran ingresar a $\mathrm{UCl}$ y aquellas pacientes que cumplieran criterios para LRA según los criterios RIFLE y AKIN. Se excluyeron pacientes con lesión renal crónica, o aquéllas con expediente clínico extraviado.

Posteriormente, se realizó una revisión minuciosa y sistemática de los expedientes clínicos enfocada a recabar: la edad de la paciente; tiempo de estancia en la UCl; diagnóstico de ingreso y de egreso (Sx de HELLP, preeclampsia, eclampsia, hemorragia obstétrica y sepsis); sobrevida; número de embarazos; edad gestacional; daño renal previo; uresis en 24 horas y resultados de análisis clínicos (hemoglobina, hematocrito, plaquetas, creatinina sérica, urea, BUN, ácido úrico, proteinuria).
Se analizó si las pacientes cumplían con criterios de lesión renal aguda según los criterios RIFLE Y AKIN. Se estimó también la gravedad y mortalidad de las pacientes de acuerdo a los criterios APACHE II. Se generó una hoja de datos como instrumento de recolección de datos elaborado en Microsoft Word 2010. Ya recabados los datos mencionados, se realizó una matriz de datos en Microsoft Excel 2010 donde se clasificó la información según las variables principales de estudio (pacientes con lesión renal aguda) y covariables (edad de la paciente, número de gestas, SDG al ingreso, diagnóstico de ingreso, tiempo de estancia en la $\mathrm{UCl}$, uresis en 24 horas, resultados de análisis clínicos). Por último, se crearon tablas de contingencia y se importaron las matrices de datos a programa IBM SPSS25 para su análisis utilizando estadística descriptiva.

\section{Análisis estadístico}

Las variables cuantitativas se analizaron según su distribución reportándose como medidas de tendencia central (media, mediana) y medidas de dispersión (desviación estándar y rangos). Las variables cualitativas se analizaron calculando la frecuencia absoluta y relativa (porcentaje). El análisis estadístico se llevó a cabo mediante la prueba t de Student. Los resultados se consideraron estadísticamente significativos si $p<0.05$.

\section{Definiciones}

La preeclampsia se define como la presencia de cifras tensionales mayores o iguales a $140 / 90 \mathrm{mmHg}$, proteinuria mayor de $300 \mathrm{mg} / 24$ horas, creatinina sérica elevada (> $30 \mathrm{mg} / \mathrm{mmol}$ ) en la gestante con embarazo mayor de 20 semanas o hasta dos semanas postparto. La eclampsia se define como una complicación de la preeclampsia severa, frecuentemente acompañada de síntomas neurológicos, alteraciones visuales, evento vascular cerebral ocurrido hasta el décimo día postparto. ${ }^{6}$ El síndrome de HELLP fue definido por los criterios de Sibai 1990, hemólisis (bilirrubina $>1.2 \mathrm{mg} / \mathrm{dL}$ o deshidrogenasa láctica > $600 \mathrm{IU} / \mathrm{L}$, enzimas hepáticas elevadas (aspartato aminotransferasa o alanina aminotransferasa > $70 \mathrm{IU} / \mathrm{L}$ ) y trombocitopenia (plaquetas < $100,000 / \mathrm{mm}^{3}$ ). La proteinuria se definió si la pérdida de proteínas en orina $>300 \mathrm{mg} / 24$ horas. $^{7}$

Investigación aprobada por el Comité de Ética y el Comité de Investigación del Hospital de la Mujer de acuerdo a los principios éticos de Declaración de Helsinki.

\section{Criterios de selección}

Criterios de inclusión: pacientes obstétricas complicadas que ameritaran ingresar a la $\mathrm{UCl}$. Pacientes que cumplieran con criterios para LRA. 
Criterios de exclusión: diagnóstico de lesión renal crónica o previa; diagnóstico distinto a complicaciones obstétricas al ingreso; pacientes con expediente clínico no disponible y pacientes referidas a otra unidad médica para su manejo.

\section{Objetivos}

Primario: Definir la prevalencia y los factores asociados a la lesión renal aguda en el embarazo (PR-AKI) en las pacientes obstétricas complicadas en la Unidad de Cuidados Intensivos del Hospital de la Mujer, Morelia, Michoacán.

Secundarios: Identificar la edad en que se presentan las complicaciones obstétricas con mayor frecuencia. Reconocer la etiología desencadenante de lesión renal aguda en el embarazo en pacientes obstétricas atendidas en la UCl. Clasificar la gravedad por grupos de las pacientes con embarazo obstétrico complicado en la Unidad de Cuidados Intensivos del Hospital de la Mujer tomando como referencia la escala APACHE II.

\section{RESULTADOS}

Se revisó un total de 213 expedientes de pacientes con complicaciones obstétricas. Se excluyó un total de 59 pacientes: cuatro casos con lesión renal crónica y 55 casos por no contar con el expediente clínico. Sólo 154 pacientes cumplieron con los criterios de selección. Ingresaron a la $\mathrm{UCI}$ un promedio de $25.6 \pm 1.6$ pacientes obstétricas complicadas por año.

Edad: la edad promedio de ingreso fue 28.53 años $\mathrm{DE} \pm 7.5$. La edad más frecuente en que se presentaron complicaciones obstétricas fue el grupo de 20-29 años con $42.8 \%(n=66)$, seguido del grupo de 30-39 años con $38.3 \%(n=59)$, el grupo de 13-19 años con $15.5 \%(n=24)$ y el grupo de $>40$ años con $3.2 \%(n$ $=5)$.

Trimestres del embarazo: de las 154 pacientes, $90 \%$ $(n=139)$ presentaron la complicación obstétrica en el tercer trimestre, $8 \%(n=12)$ en el segundo trimestre, y sólo $2 \%(n=3)$ en el primer trimestre.

Número de embarazos: se encontró que existe mayor frecuencia de complicaciones en pacientes que cursaban su primer embarazo representando $31.1 \%(n=$ $48)$. De las pacientes, $26.6 \%(n=41)$ tuvieron cuatro o más embarazos, $22.7 \%(n=35)$ eran secundigestas y, por último, en $19.4 \%(n=30)$ se trató de pacientes tercigestas. No se encontró diferencia estadística significativa entre los cuatro grupos $(p=0.19)$.

Etiología: el diagnóstico más frecuente en las pacientes obstétricas complicadas que ingresaron a la $\mathrm{UCI}$ fue la eclampsia con $36 \%(n=54)$, seguida de la preeclampsia con $35.3 \%(n=53)$, síndrome de HELLP con
$29.3 \%(n=44 p)$, hemorragia obstétrica con 19.3\% $(n=$ 29) y sepsis con $10 \%(n=15)$. De las pacientes, $94 \%$ $(n=141)$ tenían otra comorbilidad (diabetes mellitus, desequilibrio hidroelectrolítico, neumonía, obesidad o evento cerebrovascular, entre otras).

Lesión renal aguda: de las pacientes obstétricas complicadas ingresadas a la $\mathrm{UCl}, 23 \%(n=35)$ se conocía con lesión renal aguda desde el momento de su ingreso. En una segunda revisión se aplicaron las escalas aceptadas internacionalmente para LRA y se encontró que $59.0 \%(n=91)$ presentó criterios para LRA por RIFLE; asimismo, 59.7\% ( $n=92)$ cumplió con criterios por AKIN para LRA.

Se demostró que existe un subdiagnóstico de 36.7\% de PR-AKI con un valor estadísticamente significativo ( $p$ $=0.000007$ ), con escasa concordancia según el índice de Kappa Cohen (Figura 1).

Posteriormente, se realizó un análisis de las pacientes por grupos: pacientes con criterios RIFLE positivos y pacientes con criterios AKI positivos para determinar el estadio en el que se encontraban. Dentro de la clasificación de RIFLE, predominó el grado 3 con $43.9 \%$ $(n=40)$, seguido del grado 1 con $34 \%(n=31)$ y por último el grado 2 con $21.9 \%(n=20)$. Por otra parte, de acuerdo a la clasificación de AKIN predominó el grado 3 con $43.4 \%(n=40)$, seguido por el grado 1 con $34.7 \%(n=32)$ y por último el grado 2 con $21.7 \%$ $(n=20)$.

Se encontró que en las pacientes con evento obstétrico complicado, las patologías que se asociaron a PR-AKI fueron síndrome HELLP y preeclampsia $(p=0.0003$ y $p=0.01$, respectivamente). Las otras comorbilidades (eclampsia, hemorragia obstétrica y sepsis no obtuvieron $p$ significativas $(p>0.05)$ para LRA (Figura 2).

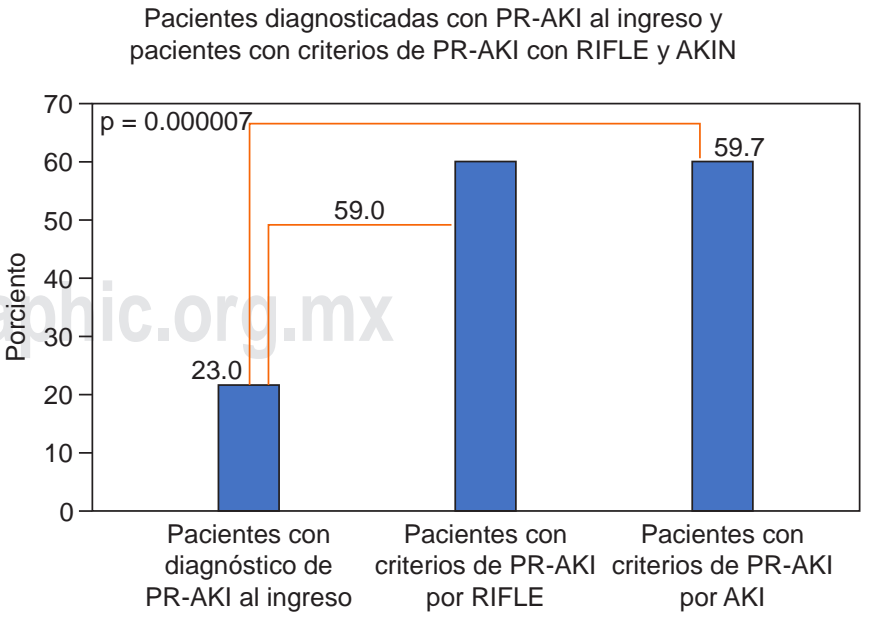

Figura 1: Pacientes diagnosticadas con lesión renal aguda en el embarazo (PR-AKI) al ingreso y pacientes con lesión renal aguda en el embarazo utilizando los criterios de lesión renal aguda por RIFLE y AKI. 
Patologías de las pacientes obstétricas complicadas ingresadas a la UCl con PR-AKI

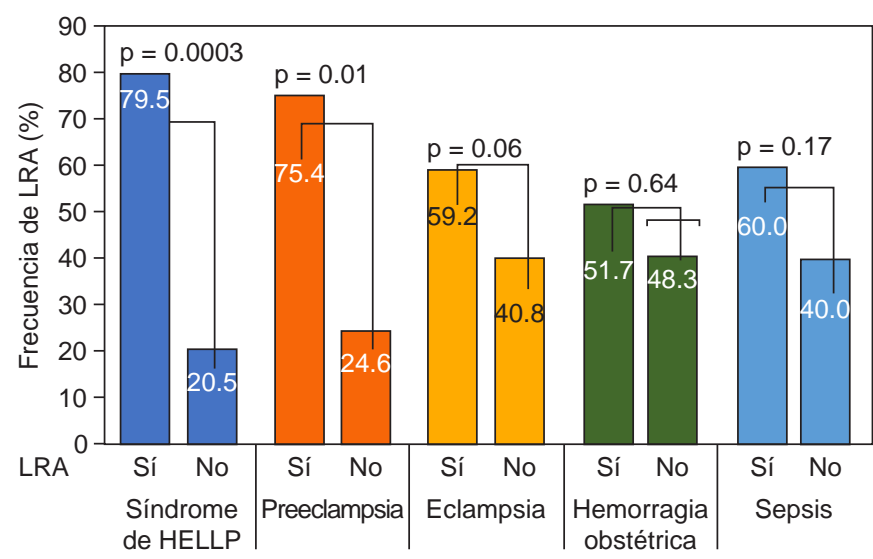

Figura 2: Patologías de las pacientes obstétricas complicadas ingresadas a la UCI con lesión renal aguda en el embarazo (PR-AKI).

$\mathrm{UCl}=$ Unidad de Cuidados Intensivos, LRA = Lesión renal aguda

Se demostró que los rangos de proteinuria no aumentaron proporcionalmente según el grado de progresión de la escala AKIN. Para un AKIN $0(n=62)$ se encontraron niveles de proteinuria en rangos de $120 \pm$ $45 \mathrm{mg} / \mathrm{dL}$; para las pacientes con AKIN $1(n=32)$ de $204 \pm 12 \mathrm{mg} / \mathrm{dL}$; para las que presentaron AKIN $2(\mathrm{n}=$ $20 \mathrm{p}$ ) de $246 \pm 33 \mathrm{mg} / \mathrm{dL}$, y para las pacientes AKIN 3 ( $\mathrm{n}$ = 40) de $238 \pm 17 \mathrm{mg} / \mathrm{dL}$.

Se realizó el análisis estadístico donde se relacionó el promedio de proteinuria de cada uno de los grupos de pacientes según los grados de AKIN (1, 2 y 3). Solamente existe significancia estadística al comparar los grupos AKIN 0 vs. AKIN 3 ( $p=0.0004)$ y AKIN 1 vs. AKIN $3(p=0.009)$ (Figura 3).

Estancia intrahospitalaria: de las pacientes obstétricas complicadas, $57 \%(n=88)$ requirió más de 72 horas de estancia en la $\mathrm{UCI}, 24 \%(n=37)$ requirió de $24-48$ horas, mientras que $19 \%(n=29)$ requirió de 49 72 horas.

Terapia de reemplazo renal: un total de 40 pacientes fueron clasificadas como grado 3 con ambas escalas (AKI y RIFLE), de las cuales solamente $20 \%(n=8)$ requirió terapia de reemplazo renal continua (Figura 4).

Las indicaciones que se consideraron para el inicio de la terapia de reemplazo renal continua fueron la elevación de azoados (elevación superior al triple del valor basal, creatinina sérica $>4 \mathrm{mg} / \mathrm{dL}$ o aumento agudo de creatina sérica $>0.5 \mathrm{mg} / \mathrm{dL}$ ) y sobrecarga hídrica (ganancia de peso corporal superior al $10 \%)$, representando cada una $50 \%(n=4)$.

Gravedad: tomando como referencia los criterios APACHE II, se asignó la puntuación correspondiente a cada paciente y se clasificaron por grupos de acuerdo al grado de mortalidad. De las pacientes, 33.1\% ( $n=$ 51) fueron clasificadas como grado 1 , seguido en fre- cuencia del grado 3 con $26.6 \%(n=41)$, el grado 2 con $16.8 \%(n=26)$, grado 4 con $9 \%(n=14)$, grado 5 con $6.4 \%(n=10)$, grado 6 con $5.1 \%(N=8)$, grado 7 con $1.9 \%(n=3)$ y por último, el grado 8 con $0.6 \%(n=1)$ (Figura 5).

Mortalidad: de las pacientes que desarrollaron PRAKI, se observó una mortalidad de $2.59 \%(\mathrm{~N}=4)$, la tasa de supervivencia fue de $97.4 \%(n=150)$. Del total de las defunciones $(n=4) 50 \%(n=2)$ presentó LRA.

\section{DISCUSIÓN}

La prevalencia de la lesión renal aguda asociada al embarazo complicado (PR-AKI) en este estudio fue de 6.7\%, mayor que lo reportado en la literatura en EUA para los países en desarrollo, ${ }^{5}$ y muy similar a lo descrito en la GPC (Guía de Práctica Clínica Mexicana) para el tratamiento de hipertensión en el embarazo de $2010(5 \%) .{ }^{6}$

Se realizó un análisis de las pacientes que fueron admitidas a la UCI con diagnóstico de LRA y todas las que cumplieron con criterios RIFLE o AKIN para LRA y se demostró un subdiagnóstico de PR-AKI de 36.7\%. En la mayoría de las pacientes se detectó en una etapa de «falla» de la escala RIFLE o AKIN etapa 3, es decir, cuando la TFG se ha reducido hasta en $75 \%$, por lo cual las medidas de nefroprotección resultaron insuficientes.

En nuestro estudio se desconocían los valores de creatinina sérica pregestacional; se sabe que en la paciente gestante la TFG puede aumentar hasta en $50 \%$, disminuyendo la creatinina sérica en promedio en 0.4 $\mathrm{mg} / \mathrm{dL}$, lo cual pudo representar un sesgo en nuestro estudio y la prevalencia de la PR-AKI podría ser aún mayor en este grupo de pacientes.

Otro aspecto interesante es que la principal etiología de la PR-AKI está asociada con síndrome de HELLP y preeclampsia al igual que lo reportado en países con un elevado nivel socioeconómico. Se demostró que la pro-

Proteinuria en las pacientes obstétricas complicadas ingresadas a la $\mathrm{UCl}$ con $\mathrm{PR}-\mathrm{AKI}$ según $\mathrm{AKIN}$

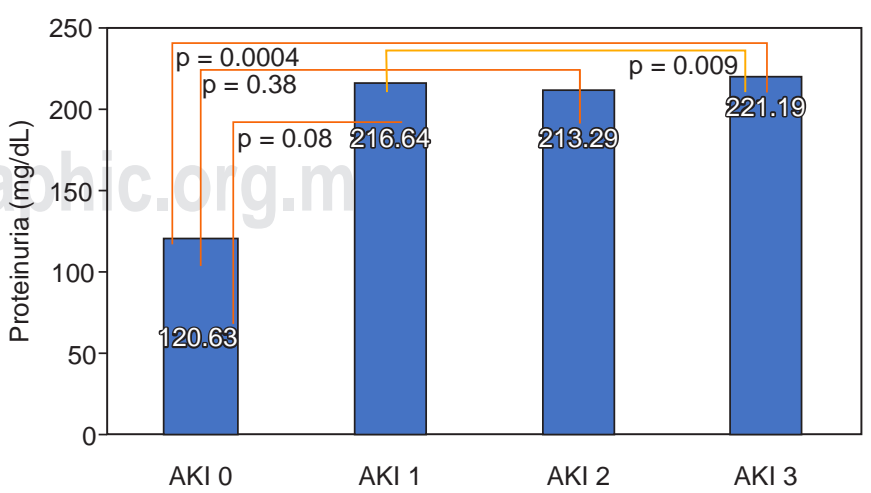

Figura 3: Proteinuria de las pacientes obstétricas complicadas ingresadas a la $\mathrm{UCI}$ con lesión renal aguda en el embarazo (PR-AKI) según los criterios AKI. $\mathrm{UCl}=$ Unidad de Cuidados Intensivos 
Pacientes con embarazo complicado ingresadas a la $\mathrm{UCl}$ con LRA grado 3 por AKIN y RIFLE que requirieron TRRC

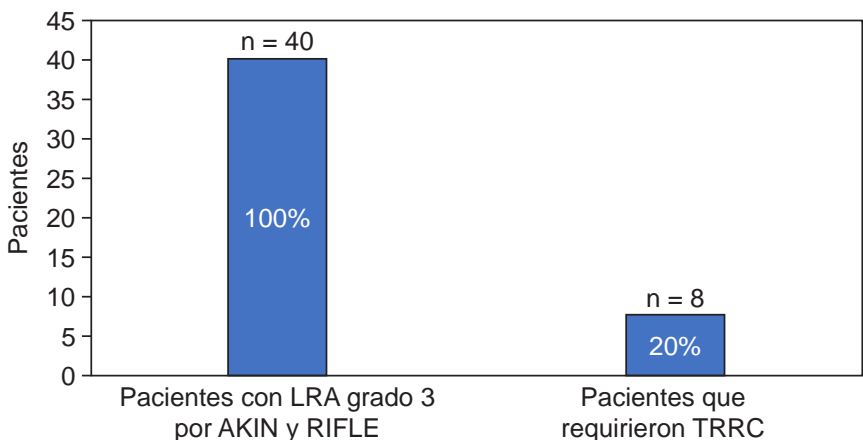

Figura 4: Pacientes con embarazo complicado ingresadas a la UCI que requirieron terapia de reemplazo renal continua en el Hospital de la Mujer. $\mathrm{UCl}=$ Unidad de Cuidados Intensivos, LRA = lesión renal aguda, TRRC = terapia de reemplazo renal continua.

teinuria no tiene una relación proporcional con el grado de daño renal.

En cuanto a los días de estancia en la UCI, $57 \%$ de las pacientes obstétricas que ingresaron permanecieron más de 72 horas. El reconocimiento temprano de PR-AKI podría disminuir la demanda y los costos de los servicios de la UCl.

En nuestro estudio, de las pacientes que presentaron PR-AKI grado 3, sólo 20\% requirió TRRC. En otro estudio estadounidense realizado publicado por Rao y Jim sólo 9\% ingresó a TRR. ${ }^{8}$ Consideramos importante que se realicen estudios posteriores para crear estrategias que permitan la detección oportuna de esta entidad y evitar la progresión del daño renal en la paciente obstétrica complicada.

La mortalidad en nuestro estudio fue baja con $2.59 \%$, menor que lo predicho según los criterios APACHE II. De las pacientes obstétricas complicadas que fallecieron, $50 \%$ desarrollaron PR-AKI.

\section{CONCLUSIONES}

La PR-AKI es una enfermedad con una alta prevalencia que afecta principalmente a mujeres primigestas entre 20 29 años de edad durante el tercer trimestre gestacional.

Las complicaciones obstétricas más frecuentes en la lesión renal asociada al embarazo son el síndrome de HELLP y la preeclampsia. La PR-AKI está subdiagnosticada hasta en $36.7 \%$.
Las pacientes obstétricas complicadas ameritan más de 72 horas en la UCI.

De las pacientes con PR-AKI grado 3, sólo $20 \%$ requirió TRRC.

En el Hospital de la Mujer de Morelia, México se utiliza la TRCC como medida terapéutica en pacientes con lesión renal severa.

El riesgo de mortalidad de las pacientes obstétricas complicadas que ingresan a la $\mathrm{UCl}$ es de $2.59 \%$.

El manejo exitoso de PR-AKI requiere un enfoque multidisciplinario con una estrecha colaboración entre nefrólogos, obstetras, médicos intensivistas y otros miembros del equipo. La identificación de la etiología subyacente de PR-AKI es crucial.

\section{REFERENCIAS}

1. Van Hook JW. Acute kidney injury during pregnancy. Clin Obstet Gynecol. 2014;57(4):851-61. doi: 10.1097/ GRF.0000000000000069.

2. Arrayhani M, El Youbi R, Sqalli T. Pregnancy-related acute kidney injury: experience of the Nephrology Unit at the University Hospital of Fez, Morocco. ISRN Nephrol. 2013;2013:109034. doi: 10.5402/2013/109034.

3. Bentata Y, Housni B, Mimouni A, Azzouzi A, Abouqal R. Acute kidney injury related to pregnancy in developing countries: etiology and risk factors in an intensive care unit. Indian $J$ Nephrol. 2017;27(2):113-117. doi: 10.4103/0971-4065.194394.

4. Carrillo ER, Castro PJ. Escala RIFLE. Fundamentos y su impacto en el diagnóstico, pronóstico y manejo de la lesión renal aguda en el enfermo grave. Rev Asoc Mex Med Crit y Ter Int. 2009;23(4):241-244.

5. Sibai BM. The HELLP syndrome (hemolysis, elevated liver enzymes, and low platelets): much ado about nothing? Am J Obstet Gynecol. 1990;162(2):311-316.

6. CENETEC. Detección, diagnóstico y tratamiento de enfermedades hipertensivas del embarazo. (1 de Enero de 2017) Recuperado el 28 de Mayo de 2019, de CENETEC: http:// www.cenetec-difusion.com/CMGPC/IMSS-058-08/ER.pdf.

7. Ali A, Ali MA, Ali UM, Mohammad S. Hospital outcomes of obstetrical-related acute renal failure in a tertiary care teaching hospital. Ren Fail. 2011;33(3):285-290.

8. Rao S, Jim B. Acute kidney injury in pregnancy: the changing landscape for the 21st century. Kidney Int Rep. 2018;3(2):247257. doi: 10.1016/j.ekir.2018.01.01.

Patrocinios: Ninguno.

Relación de conflicto de intereses: Los autores declaran no tener conflicto de intereses.

Correspondencia:

Dr. Alfonso Estrada Gutiérrez

Guillermo Roquet Núm. 250,

Poblado Ocolusen, 58270

Tel: 443273-4621

E-mail: estragua@hotmail.com 